\title{
Cooperative Community Opportunities and the ADS
}

\author{
Donna Thompson ${ }^{1, \star}$ and Carolyn Grant ${ }^{1}$ \\ ${ }^{1}$ Harvard-Smithsonian Center for Astrophysics
}

\begin{abstract}
The ADS is used daily by the astronomy community and benefits from the cooperative nature of our contributors. In this poster we will outline some of the ways that the library community can continue to contribute to this resource. As online publishing trends continue to evolve, conference proceedings and presentations, $\mathrm{PhD}$ theses and other digital artifacts are being deposited to research data repositories such as Zenodo or Dataverse. We will describe the process that ADS follows to include relevant collections from such repositories when properly curated by librarians or researchers. In addition, while most of what we receive is vetted before it gets to us, we sometimes find ourselves in the position of having to determine whether or not an article, proceeding or other submission is refereed. ADS has guidelines on how we determine this but sometimes the lines are not so clear and sometimes the arguments on both sides are compelling. The process that we follow will be outlined and we would appreciate feedback on this topic. Finally, given the expertise of librarians in their field and within their organization, the ADS encourages librarians to consider submitting curated institutional bibliographies for inclusion in the ADS.
\end{abstract}

\section{Introduction}

The ADS seeks to provide comprehensive, but not all-inclusive, coverage of the fields of astronomy and astrophysics. To this end we welcome contributions to our collection that we may have missed and value added curation from the library community. In this paper we will describe some of the issues that we routinely face and the content which we would welcome.

\section{Missing content}

We receive regular feeds from both major and minor publishers, scientific societies, and other data providers such as CrossRef. We regularly monitor lists of conferences and check for their publication online or in print so that we can add this information to the ADS corpus. We encourage the ADS user community, particularly librarians, to contribute to our collection by communicating with us when gaps in our coverage are detected. One part of the collection where we know we are missing entries is the $\mathrm{PhD}$ theses. As many universities are no longer depositing theses into data depositories and opting instead to add them to their institutional archive, ADS no longer has an automated source for this valuable collection. We encourage submissions from both US and international institutions. We are happy

^e-mail: dthompson@cfa.harvard.edu ORCID: 0000-0001-6870-2365 
to work with individuals to make this process streamlined on both sides. We download records from a select list of Zenodo collections on a weekly basis and welcome your suggestions for communities and topics to add to the content harvested by the ADS. These collections are typically contents of a conference proceeding which have been vetted by the organizer or other affiliated participant. Because Zenodo uses the Open Archive Initiative protocol (https://www.openarchives.org/pmh/), this allows additional records to be added after the initial batch has been submitted, which enables us to automate improving coverage of these proceedings. Additionally, we harvest the collection of astronomy $\mathrm{PhD}$ theses from Zenodo. Dataverse is a world-wide project designed to allow researchers to deposit data into open source archives on their own computer or computers at a publisher or institution. Dataverse links referenced in the astronomy literature can be included in the ADS by sending us the appropriate linking data.

\section{What we include}

We frequently receive requests from journal editors or users to add a certain journal title to the ADS. We verify that the publication is appropriate for inclusion by examining the journal for its relevance to ADS, including relevance to the scientific study of astronomy; content in any of the subfields of astronomy or physics; non-astronomy content of interest to astronomers in different subfields; and content in related fields that has astronomical relevance.

In addition, we expect journals to have:

- A quality and scope of interest to an international audience

- An ISSN

- A CrossRef registration

- Standard conventions for scholarly journals

- An informative title and descriptive article titles

- Full contact information for authors and editors

- Usable metadata, including abstracts, titles, and key words

- References that are current, from quality journals, and of an appropriate number for the article in a standard format

- All or mostly all original articles

- Articles that are written by professionals in their field.

- Publication on a regular schedule appropriate for the established frequency of the journal Electronic-only journals must:

- Provide evidence of archiving arrangements, online or otherwise

- Include a posting date for articles and revisions

- Have published at least three issues

Features that support acceptance:

- Sponsorship by an astronomy or physics association or other society

- Astronomers or physicists among editorial board members and authors

- Indexed in other scholarly databases

- Online access 
- English table of contents

For e-journals:

- Presence of version number

- Indication of where previous versions can be obtained (Links)

For a full list of our evaluation criteria see the FAQ section linked from the ADS search page.

We have created these metrics for evaluation in an effort to weed out journals which are predatory by nature. Journals which will publish any article for a fee, or reprint existing articles for additional exposure compromise the integrity of the data content in the ADS.

\section{Refereed or not}

Occasionally we receive an email from a user stating that his or her paper is refereed and why is it marked as non-refereed in the ADS? ADS considers articles refereed when they appear in journals that participate in peer review. Peer review is the process of having articles reviewed by experts in the field before publication. ADS staff check journal websites and author instructions to verify the peer review status. Sometimes periodical directories are consulted, such as Ulrich's International Periodicals Directory.

Non-refereed materials, such as conference proceedings, circulars and bulletin entries, are screened only by an editor and not sent out for peer review. The ADS does not consider materials that are verified only by an editor as refereed.

We actively collaborate with other information providers to improve the rubric for determining what qualifies as "refereed". We closely follow industry standards as they evolve through projects such as CrossMark (https://www.crossref.org/services/crossmark/), which can allow specification of metadata about the conference such as acceptance rate of papers and type of contributions which can help in such decisions.

\section{Institutional bibliographies}

ADS is used by many librarians to curate their institutional bibliographies. The API available through ADS-beta version can help to streamline the process (see the poster Managing Institutional Bibliographies using the ADS API: A new workflow using Google Sheets? from this conference for an example.) ADS can incorporate curated bibliographies into our holdings and add a filter for individual institutions. This will make it easier for ADS users to correctly identify researchers at each institution. By providing the ADS with an institutional bibliography we can create a "bibgroup" for that institution which will be entered into our list of filters and can be used easily by users to filter papers. This is one way to make author disambiguation easier.

Another effort to streamline author disambiguation is allowing users to search by affiliation, which has been implemented in its basic form in ADS-beta. Improvements to the basic affiliation search are underway, including normalized organization names and the ability to filter on the results.

ADS has partnered with ORCID to allow users to claim papers and associate the papers with an author's ORCID through the ADS. Users have the capability to claim papers in the ADS, have those claims uploaded to ORCID, and to search by ORCID id in ADS-beta. With the proper permission settings, users can grant ADS permission to track claims and enable other users to find one's articles more easily. More details about this can be found at http://adsabs.github.io/help/orcid/claiming-papers.

ADS is planning to integrate ORCID mappings curated by librarians into its database to complement data provided by publishers and claimed by users. A trial using data from the HarvardSmithsonian Center for Astrophysics is planned for later this year. 


\section{Conclusion}

The ADS is used by members of the astronomy community and the general public on a daily basis. We are continuously updating the data and metadata and will gladly accept verified input from community members. If you have or know of any relevant conference proceedings, journals or $\mathrm{PhD}$ theses to add to our collection, please contact us to see how we can incorporate the data. We can be reached by email at adscfa.harvard.edu or through our Feedback forms available from any search page on ads.harvard.edu. 